Trans youth and social media: moving between counterpublics and the wider web

Olu Jenzen *

School of Media, University of Brighton, Brighton, UK

Watts Building Lewes Rd Brighton BN2 4AT

United Kingdom of Great Britain and Northern Ireland

o.jenzen@brighton.ac.uk

Ackn: Y

CN: Y

Word count: 7296 


\title{
Trans youth and social media: moving between counterpublics and the wider web
}

\begin{abstract}
Today's trans youth grew up with the internet and online LGBTQ resources and spaces are important to these communities. This article focuses on conceptualizing the digital cultural strategies that trans and gender questioning youth adopt both as social media users and producers in order to cope and thrive. Drawing on ethnographic data detailing a group of trans youth's engagements with LGBTQ social media counterpublics and the wider web, and their movement between these spheres, in combination with close readings of online material identified as salient by the participants, this article argues that in the face of rampant transphobia and cis coded online paradigms, trans youth respond both critically and creatively. More specifically, I highlight how they resist prescribed user protocols of mainstream social networking sites as well as employ pragmatic strategies for navigating a binary gendered online world, staking out their own methods and aesthetics for self expression and community formation. Having examined the content and style of social media examples highlighted by the participants, the article contends that trans youth's consumption and production of types of online and social media is significantly more diverse than research to date has recognised.
\end{abstract}

Keywords: LGBTQ; LGBTQ Digital Culture; LGBTQ Youth Activism; Social Media; Trans Youth.

\section{Introduction}

Facebook is a pub where Tumblr is more like a chi chi bar...

What does that make Reddit?

Reddit is a club. Everyone is there, nobody knows each other, they all just sort of exist. (Transformers workshop 10.4.2015)

Online digital culture and social media are ubiquitous in young people’s lives. For many young people, the internet is completely embedded within the way they think and live. This article focuses on conceptualizing the digital cultural strategies that 
trans and gender questioning youth adopt both as social media users and producers in order to cope and thrive. The term trans is used here to represent a broad range of gender identity variances and subcultural collective identities that have relevance to the young people who participated in the study, thus denoting 'not only identities such as transgender, transsexual, trans man, and trans woman [...] - but also identities such as genderqueer, neutrios, intersex, agender, two-spirit, cross-dresser, and genderfluid' (Tompkins 2014, 27). Drawing on ethnographic data detailing a Brighton (UK) based group of trans youth's engagements with LGBTQ social media counterpublics and the wider web, and their movement between these spheres, in combination with close readings of online material identified as salient by the participants, this article argues that in the face of rampant transphobia (e.g., The House of Commons Transgender Equality Report 2016; Transgender Europe 2009-2016) and cis and straight coded online paradigms, trans youth respond both critically and creatively. More specifically, I highlight how they resist prescribed user protocols of mainstream Social Networking Sites (SNS) as well as employ pragmatic strategies for navigating a binary gendered online world, staking out their own methods and aesthetics for self expression and community formation. Having examined the content and style of digital and social media examples identified by the participants, the article contends that trans youth's consumption and production of online and social media is significantly more diverse and multi-layered than research to date has recognised.

Previous research has predominantly focused on media representations of transgenderism yet few studies have investigated trans identifying audiences (Capuzza 2016; Cavalcante 2013 and Kosenko et al. 2016) and their consumption and production of social media content. In their 2003 article on lesbian, gay, bi and trans (LGBT) youth in the US and the UK, Ryan and Rivers note that LGBT youth are 
increasingly visible in society and that this development has also brought increased victimization. In the time since this research was undertaken the visibility of transgender youth has become increasingly mainstream on television and film and in the popular press. Transgender and gender questioning youth nevertheless remain marginalized and often experience social isolation (Grossman and D’Augelli 2006). Furthermore, because trans youth 'violate conventional gender expectations', Grossman and D’Augelli (2006, 112) contend, this group often become victimised and disciplined for this transgression in the form of bullying, discrimination and abuse, leading to them experiencing 'more psychosocial and health problems than other social groups' (ibid). A particular concern for trans youth is the routine and institutionalized practice of denying them their own understanding and articulation of their gender. Non-conforming teenagers in particular may experience this acutely, as they have not yet fully gained 'adult' social or economical independence and thus have limited agency in terms of social gender role expression.

Nevertheless, as Jones and Hillier (2013) have shown, trans youth demonstrate the capacity to respond to social rejection and discrimination through activism, and in their study 'many [trans youth] held high hopes for the ways in which they might impact their worlds in the future' (287). Against this background, arguably, LGBTQ online resources and spaces are important sources of information and socialization for trans youth (Jenzen and Karl 2014). Research indicates that LGBTQ youth typically spend more time online than youth in general (GLSEN 2013). Various studies (Driver 2007; Laukkanen 2007; Pullen 2014) indicate that LGBTQ youth go online and seek out LGBTQ-related content for a sense of belonging and to socialize. To many young people it is also the main space for political expression and activism. As Carah and Dobson (2016) point out, social media afford groups who rarely are in a position to 
influence broadcast or other traditional media more control over how they are represented. Yet mainstream SNS, such as Facebook, are carefully managed spaces of civility with clear commercial imperatives and users are increasingly up against restricting limitations. In what follows I will tease out how the trans youth in the study navigate, resist and respond creatively to such limitations.

My enquiry posits that trans youth are ideally positioned to 'consider the impact of transgender representation on themselves and their peers' (McInroy and Craig 2015, 606) and will outline how young people articulate their critique of the cisnormativity (Lennon and Mistler 2014) of the internet and mainstream media and look at their motivations for turning to alternative platforms and user generated content. Lastly, the article considers the audio-visual affordances of some online digital media as key components that enable particular modes of meaning making that are intensely valued by trans youth.

\section{Exploring trans youth online cultures}

In this empirically informed article that draws on ethnographic research to delineate both constricting and enabling structures and affordances of online digital and social media from the point of view of trans youth, I adopt a mixed methods approach that combines close readings of online audio-visual and textual material (YouTube vlogs and other videos, Tumblr microblogs, memes, online comics etc.) with community based collaborative research. The two methods are related in that the participants from the Transformers workshop have selected the online material dealt with in the media analysis. Close readings of user generated content, I posit, are vital if one wants to understand the 'specific formal strategies' (Horak 2014, 573) employed in trans youth digital media. 
The ethnographic data was generated through a creative workshop with participants aged 16-26 who regularly attend the fortnightly Transformers group, a social safe space for gender exploring youth at Allsorts Youth Project, Brighton, UK, supported by youth workers (see www.allsortsyouth.org.uk). Ethical approval for this work has been granted by the University of Brighton. No specific demographic data about participants was collected. The age range of participants is based on the organisation’s own group stratification. Participants’ consent to collect data was confirmed in writing.

Brighton has a vibrant LGBTQ community and has been dubbed UK's 'gay capital', which has both positive and challenging implications for the local trans community (See Browne and Bakshi 2013). LGBTQ youth services are nevertheless high in demand. Eight young people who attend the Transformers drop-in group participated in a 90 minute long informal creative workshop that was recorded as a group interview. Participants also had 1-2-1 breakout conversations with the researcher that were recorded using note taking. The workshop was open to everyone who attended the drop-in session and participants self selected to opt in to participate in the data collection. Care was taken to design activities that were directly beneficial for the participants and fitted the ethos of the their regular meetings. We used a range of creative craft methods to facilitate discussion and reflection on topics such as trans online digital media, health, community, culture, and everyday life in a fun and accessible way that didn’t require any particular skills. Outputs included creative idea sketches, stories, drawings, and collages, covering topics such as celebrities, fan communities, and experiences from a cis-biased school environment to mention a few. These were also recorder as data. In addition iPads were used to facilitate discussions about online material in an informal show and tell style conversation. 
Some participants thus engaged the researcher in a 'walk through' of online material and pages were bookmarked or captured using screen shots. In this way participants were free to map online 'journeys' according to their own priorities, interests and readiness to share. These journeys took us through a wide range of web pages they regularly sought out, such as various online comics and fan forums; the social news website Reddit.com and multiple subreddits; Tumblr blogs, and YouTube. The show and tell style resulted in naturally developing conversations, incorporating the exploring of hyperlinks and related materials as we came across it. Care was taken not to capture any personal content. The sample of vloggers this article examines is thus not arbitrary but exclusively based on the young people's selection. As this article illustrates, such youth led enquiries produce completely different stories than those that are researcher-focussed or guided by platform-specific metrics, such as highest numbers of followers, shares or views etc. The analysis pays particular attention to critical areas foregrounded in the ethnographic material: the affordance of the networked media technologies as technologies of both the self and the community; digital counterpublics and opportunities for everyday activism.

\section{A cis-normative internet}

There can be little doubt that the internet has offered a 'significant technological transformation for transgender individuals and communities' (Cavalcante 2016, 113). However it is important to recognize that whilst online participation can be enabling of self-expression and community building in significant ways (Kosenko et al. 2016), mainstream ideologies also dominate online culture. Trans youth’s experiences of mainstream digital media show how the internet is not only 'coded' cis in a discursive sense, but also in terms of information management. Similarly, digital networks are 
also racialised and, as Nakamura (2013) points out, the capability of internet racism is inescapable.

The harmful impact of traditional media representation on transgender people is well documented (Trans Media Watch 2010). And although news coverage on trans matters and lives has expanded, it has simultaneously 'constructed and reconstructed a normative definition of "transgender”' (Capuzza 2016, 84). This resonates with what participants in this study express about struggling to find the normative and narrow range of trans identities and experiences represented by mainstream media meaningful. Mainstream media continues to sensationalise trans lives and bodies, whilst important issues such as 'employment and legal, health and safety challenges' rarely make it into the news (Capuzza 2016, 91). Relatedly, a workshop participant commented that:

[the] people who really need positive support are those not yet part of the community and they are not helped by mainstream media. They just see all the crap, the negative stuff that the [mainstream] media show.

In other words, without the subcultural knowledge about how to navigate information online, young people are by default referred to ciscentric content because it is prioritised by search engines and ‘endorsed by digital gatekeepers’ (Baker and Potts 2013, 188). This shows how dominant digital mechanisms reproduce ciscentric and negative representations and contribute to trans marginalisation.

In light of this it is perhaps not surprising to find that trans youth expressed a distrust of mainstream platforms, commercial search engines and mass media. One interviewee stated: 'trans always seems to be a negative - it almost seems a negative word in itself'. Furthermore, participants offered critique of media sensationalism and hegemonic perspectives that validate cis and heteronormative concerns not just on 
trans issues but on representations of race, gender, sexuality and bodies more broadly. They attribute this to systemic structures such as the underrepresentation of transgender people in media industry positions that matter. As one participant puts it:

Unfortunately, the newspapers are run by cis white guys. [---] there are trans journalists who write for the mainstream newspapers [...] but, you won't see it on the front page because it is the editors who decide and ... Until someone who isn't cis gender [...] becomes quite high [in the management hierarchy] you won't get that.

In a similar vein Baker and Potts (2013) have critiqued search engines and the perpetuation of stereotypes via their auto-complete function. For trans youth, navigating between counter publics (or online networked communities made up of engaged and compatible individuals) and the wider World Wide Web is fraught with contradictions and frustrations. The participants' strategies for dealing with the tension between these are reflected in a popular YouTube video blog by trans identifying vlogger Uppercase Chase 1 who warns against looking up 'transgender’ on the Google search engine. 'I think Googling trans things is probably one of the worst ideas if you don’t know how to look properly' he states, thus indicating that a critical awareness of the dominant structuring of information through search engines forms a crucial part of trans youth resilience. This speaks to the coping strategies trans youth have to develop to protect themselves from being overwhelmed by harmful trans-phobic messages.

The young people's highlighting of problems around search engines chimes with DeNardis and Hackl’s (2016, 753) argument that debate and conflict around sexual politics and LGBTQ rights in particular 'are increasingly materializing within technical functions of internet governance and architecture rather than at the surface level of content'. Their examples include both the Russian and US governments’ 
direct attempts to block access to LGBTQ sites, but also more subtle forms of filters such as commercially developed 'net safety software' (e.g. the much criticized Kiddle), as well as search engines’ opaque algorithms. Researching the interaction between users' enactment of visual regimes and algorithmic dimensions of social media Carah and Dobson conclude for example that the gendered gaze 'appears to be iteratively translated into protocols and procedures of algorithmic media’ $(2016,9)$. Although their case specifically concerns images of young female bodies in mainstream nightlife promotion on social media platforms and the production of normative femininity, the processes they describe apply also to other online spheres, and there is a correlation here to what Raun (2016) notes about racial and ethnic differences in terms of the attention a trans vlogger gets and such bias means white US/ European trans vlogs get more exposed and viewed. Algorithms may not be capable of identifying cis or white bodies in the social media flow of images per se but are definitely able to determine users' behaviour and affect in relation to images and therefore work to amplify the social behaviours that value certain bodies and not others, in line with dominant norms. In other words, computer code 'uses certain representations of the world (variables and data) to produce new value' (CheneyLippold 2011, 167). On the other hand, Cheney-Lippold’s work on algorithmic categorisation practices explains that not only do the statistical models used by the web analytics to determine users' gender (and other demographics) operate according to specific 'cybernetic' forms of gender stereotyping, this algorithmic gender construction also 'defines the actual meaning of gender, class, or race themselves' (165). This clearly ingrains normative constructions - such as an understanding of gender as distinctly binary - yet also potentially opens up for a queering of gender as 
the computerised categorisation and coding practices continually and rapidly give rise to new practices.

Given that 'engineered limitations of particular [media] technologies can be as coercive as human norms of conduct' (Roth 2014, 2116) it is not unfounded that trans youth, as found in this study, take a critical view on how private internet companies mediate LGBTQ content and remain sceptical towards the dominant online public sphere. However despite built-in constraints LGBTQ youth demonstrate pragmatic approaches to their use of mainstream SNS such as Facebook as well as creative forms of resistance to the prescribed use of the social media platform. Therefore I argue that we need to look at how users negotiate - and sometimes subvert - the values and norms that technologies incorporate in order to make pragmatic use of mainstream platforms for exploring issues around gender, sexuality and identities through activism and community formation.

A good case in point is the Facebook status update widget created by Allsorts youth advocacy group Young People’s Voice in 2013 designed to incorporate your profile picture in a political statement (see fig. 1). Small-scale grassroots campaigns like this predated with quite some time, Facebook's own development of similar features such as their rainbow flag filter to celebrate pride in June 2015. Although Facebook over time has adapted to, and to an extent incorporated, these types of expressions originating in user creativity, at the time it certainly wasn't sanctioned. Whilst the widget campaign does not amount to a new kind of electronic civil disobedience, it is still a subversion of the formal conditions of expression on a particular platform. 
This person is standing up in the fight against HOMOPHOBIC, BIPHOBIC

AND TRANSPHOBIC

BULLYING

This week is Anti-Bullying Week. Please share to stamp out LGBT Bullying

Figure 1. Allsorts Facebook widget.

\section{Not on Facebook}

SNS are technologies of both the self and the community. These are spaces where we see the formation of LGBTQ youth communities through mediated communication in conjunction with the construction of LGBTQ identities through popular discourse. Whilst we currently see a clear diversification trend within young people’s social media use, Facebook remains the most used social media site with UK and US youth in general (see Lenhart 2015). For trans youth, for many of whom self-determining legal gender is still out of reach and who often struggle to be known by their preferred name, one’s Facebook profile can be an important place for both playing with and asserting one’s gender identity. One research participant described how it was an important moment to them when they changed their name on Facebook. It was primarily a way of confirming their preferred name as most friends knew them by this name already. But it did also offer a method of letting people know about their trans identity in a more public way. People in their circle responded by messaging them asking 'what does this mean?' which they seized as an opportunity to have important discussions about identifying as trans.

However, workshop participants, as noted earlier, expressed a preference for the more open-ended and visually-orientated Tumblr. They also provided an insight into the nuances of online diversification, by explaining that they found Facebook 
useful for connecting with their geographically and socially more immediate peer groups whilst Tumblr was seen as the better tool for connecting with a wider community without regard to geographic proximity, as illustrated by the vignette on the first page of this article. Another participant comments: 'I'm still on Facebook but Facebook is more local. [...] Facebook is what my mum reads, Tumblr is not'.

The appeal of Tumblr for marginalised youth is also to do with its function as virtual counterpublic. Michael Warner $(2002,119)$ describes counterpublics as discursive spaces that enable marginalized groups' articulations and 'interpretations of its members' identities, interests, and needs' in opposition to a dominant public. Cavalcante sees these as 'architectures of organized care and concern, that facilitate transgender identity work and everyday survival’ (2016, 109; see also Shapiro 2004). Because of the overwhelming cis-bias of mainstream media and publics, both Dame (2016) and Cavalcante (2016) point to the importance of 'counterpublics' (cf. Renninger 2014 and Warner 2002) for the trans community.

Tumblr, a microblogging platform founded in 2007, can be identified as a prime example of one such online counter public. Users can publish both textual and visual material including moving images, in short formats, which form a blog. Users can publish their own original material, curate other internet material, or re-blog other Tumblr posts. The site's social networking function facilitates following other bloggers or exploring subjects through tags, as well as engaging in dialogue with others via direct comments on their posts or via the question function. In contrast to Facebook's highly structured framework for setting up and maintaining a user profile, ‘identity construction’ on Tumblr is more fragmented (Oakley 2016, 2).

Trans youth also expressed a preference for Tumblr because of its visual emphasis and because of a notion of being able to find and connect with a trans 
community on this platform. Several research participants take an active role on Tumblr, motivated by the idea of making a contribution to the online community. One interviewee comments:

[on Tumblr] I do reviews and articles on LGBT subjects, particularly trans [...]. I try to offer support and basically say things that need to be said. [---] I like the idea of people feeling good when they read my stuff.

Participants active on Tumblr also have a sense of entering into conversation, not just with an imagined community but with an audience capable and willing to enjoy and respond to them. This is reflected in one participant's comment that their Tumblr style is 'satirical'. In other words, they have adopted a stylistic position that is both playful and sophisticated in terms of form.

Furthermore, as Renninger (2014) and Hart (2015) note, Tumblr blogs are less tied to 'singular identities' (Hart 2015, 204) than other SNS which may resonate with youth resisting linear narratives and essentialist conceptualisations of gender and sexual identity, whilst on the other hand complicating notions of community. It is not just that we can find 'radical pockets' of queer and trans youth output on Tumblr, the site’s DNA, or code, its collage style in combination with its ‘instability and idiosyncrasy’, Fink and Miller argue $(2014,614)$ makes it particularly conducive for non-reductive representations of daily aspects of trans lives.

As several social media researchers have pointed out, SNS are 'rhetorically and politically constructed' and their code and affordances affect both the uptake (demographically) and type and style of content shared (Oakley 2016, 6; see also boyd 2011 and Gillespie 2010). In the case of Tumblr its tagging function offers trans users 'the unique possibility to both unobtrusively make their transness relevant in personal, quotidian moments while also rendering their self-narratives more complex' 
(Dame 2016, 30) and constitutes as Oakley (2016, 6) suggests 'an excellent example of affordances shaping usage'. For LGBTQ youth in particular, the tagging practice is a welcome opportunity to self-define and manage their self-presentation. This is mirrored in the Transformers group ethos and is significant because, as Dame notes, 'their tags are their ontological stake’ (2016, 31), something young people are routinely denied in other areas of their lives.

However, whilst this affordance potentially has a lot to offer in terms of rethinking sociability and networked connections beyond traditional membership structures based on less fluid identity categories (such as LGB or T) it does not necessarily produce a ‘safe’ space or keep out transphobic or exploitative content from a user's digest of posts. Dame (2016) cites the example of pornographic material fetishizing transgender bodies being mixed in with more community-orientated posts because of using the same \#transgender tag. However it should be noted that what some users experience as a form of 'tag spam' others value as a form of 'randomness' they find liberating and regard as an integral part of the platform's functionality (see Fink and Miller 2014).

Relatedly, during the Transformers group discussion the topic turned to trans youth suicide notes on social media, which participants noted was not an uncommon thing. An example they were all familiar with is the suicide note of Leelah Alcorn, a transgender teenager from Ohio US who ended her own life in 2014, having being subjected to so called conversion therapy, a controversial and harmful practice. One participant comments:

That whole Leelah Alcorn thing has become ridiculously scary. [These are] not just trans suicides like, but trans suicides that follow a pattern, i.e. social media suicide note, and then suicide with public hashtag. That pattern - eight people if I'm up to date - in three months, [---] have [taken their lives]. 
The virtual spread of such posts, also facilitated by hashtags, they worry, could have a negative impact on other trans youth, demonstrating that for someone who is in a difficult place in terms of dealing with rejection or oppression or in in terms of their mental state, the same platforms and their affordances might further engrain selfharm.

Another key finding is the diversity and abundance of the range of social media and online forums LGBTQ youth engaged with, upon which existing research does not reflect, due to a narrow focus on mainstream SNS and commercial giants and a tendency to be directed by the user data generated by the sites’ own analytics tool. Several interviewees pointed out how they explored issues around trans identities and queer sexualities primarily through subcultural online community websites such as Fur Affinity (a fan forum with an interest in animal fantasy writing and art), trans community subreddits on Reddit, Sherlock fan fiction or online comics. Whilst furry fandom and other types of online fan communities offer both creative opportunities for queering mainstream popular culture and exploring alternative world views (see Dhaenens 2012), the appeal of the comics seems to be more about their quotidian style and use of humour. Examples mentioned by the young people include Validation (by Beranek and Crawford), Questionable Content (by Jaques) and Xkcd (by Munroe), Minority Monsters (by Kimpton) and transgirlnextdoor.com (by Wu). Some of these comics, like Validation or transgirlnextdoor, offer representations of every day life where trans characters and experiences are at the foreground but treated in a non-dramatic and incidental style and fully integrated into the world of the comic. Minority Monsters on the other hand is more camp in style and also include a wide range of mythical characters such as 'Sir Fabulous the Bisexual Unicorn', 'Queen Ravishment the Polyamorous dragon’ and ‘Captain Sashay the Genderqueer 
Merperson'. These comics are accessible to young people because they offer, in short instalments and with a visual emphasis, ways of tackling serious issues in a playful, light-hearted and humorous way.

So from this we can conclude that trans and queer youth subcultures flourish on Tumblr and YouTube but are by no means limited to these sites. We need to acknowledge this diversity in research and I argue that, for participants in this study, comics, music and various forms of online popular culture fan forums are equally important for their engagement with issues of identity and trans culture. This might provide a valuable insight for youth service providers and community outreach work. Such organisations are likely to have a web presence - often in the form of a static website or a Facebook page - but often rely on the assumption that the LGBTQ vocabulary and linguistic and visual discursive frames of reference are the prime hallmarks that are going to guide prospective users towards them. However, as the present findings suggest young people’s navigating of online forums and social media is complex and diverse, and possibly not purposeful in anticipated ways. For example, one participant who said they mainly post on trans matters on Tumblr describes herself as a 'modern strange weird hippie girl who writes like a modern strange weird hippie girl and modern strange hippie people like my style', indicating a sense of networked connections where the LGBTQ identification, subculture or vocabulary are not the primary points of recognition or connection. The interview findings presented here thus challenge the cis-centric assumption that trans youth access trans culture predominantly via LGB culture. Being aware of how online popular culture and subcultures of taste provide valuable environments for youth learning about and talking to others about identities, gender, relationships, bodies, sex and intimacy, attitudes and so on, can only enrich service provision. 


\section{Trans youth and vlogging}

Trans vlogging is a growing genre on YouTube. Recent works by Laura Horak (2014) and Tobias Raun (2015; 2016) are breaking new ground by specifically considering trans vlogs, their styles, narrative and audiences. Both Horak and Raun come to similar conclusions - that 'these videos are enormously productive for the trans youth who make and watch them' (Raun 2016, np) although they emphasize different reasons for this.

What the DIY trans videos demonstrate is how keen trans youth are to utilise the full audio-visual affordance of online digital media. Video diaries and montages are an integral part of the support structures trans youth build for themselves. This section demonstrates the significance of such mediated embodiments of trans experiences and imaginings. The majority of existing research on trans politics and culture considers, as pointed out by Raun (2015), a text based internet. Kosenko et al. (2016, np) note however that in their study 'YouTube was specifically referenced by several participants as having utility for sensemaking by providing visual representations of like-others talking about their transgender experiences'. Raun's research is mainly concerned with autobiographical video blogging. This is an established YouTube genre, which includes a set of subgenres. For example, comments from the youth I interviewed suggest that group members regard what they call ‘famous' trans vloggers’ output as primarily serving the purpose of educating non-trans audiences. This differs from the 'everyday' or peer vloggers whom they see as addressing their own community more directly and whose videos they deem more relevant to trans youth themselves. These vloggers typically reflect grassroots politics and a DIY approach to gender expression. In terms of style and content they are more 
spontaneous, both in how their videos are produced and in their repertoire of more quotidian topics.

However, what is perhaps more important is the depth and range of meaning making processes trans youth engage with through trans vlogging on YouTube. For example, many trans vloggers record bodily changes as they start taking hormones or initiate a transitioning, in a detailed video diary, often also continuously reflecting on their 'state of mind' (Raun 2015, 368). The videos record audio-visually physical changes, style changes, demeanour, body language and voice. Young people find these videos encouraging and informative; they provide testimonies of what it possible and insights into the challenges along the way. However, for some trans youth this subgenre of vlogging emphasises too heavily a transnormative narrative of a 'successful' gender reassignment, reflected in one interviewee's comment that many trans vloggers who have been documenting their transitioning timeline on YouTube tend to 'disappear' from the site once they have fully transitioned. There is nevertheless a captivation with transgender journeys of various forms among the YouTubers and their audiences, exemplified by vloggers like Isabella Bunny Bennett's posts with headings such as 'MtF VBlog \#1 - First Day of Meds', MtF VBlog \#2 - 1 month and 1 week on Hormones’ etc. More playfully approaches also exist, such as vlogger Uppercase Chase 1's fast montage of 2194 selfies in his video 'Every Day for Six Years FTM Transgender’ documenting his transition in a hypercompressed temporality, but also ‘evidencing' or marking his existence every day, day by day. Many vloggers also comment on the emotional investment they place in this digital technology, highlighting how it functions as a tool for recording or evidencing the self, for self-reflection, for mediating one’s advocacy and helps breaking out of isolation. 
Among the Transformers interviewees the most popular trans vloggers were those who are approximately the same age group as the participants. (It should also be noted that the group was predominantly white as were the majority of the trans vloggers they mentioned and unlike age, whiteness is rarely 'labeled or addressed' (Raun 2016, np) and thus as Raun points out functions as an invisible universalizing factor in the vlogs.) These young vloggers address a range of topics (in a variety of styles) such as relationships, toilets, school, family, depression, gender dysphoria, social networks, clothes, and experiences at the GP. UK based vlogger Alex Bertie provides a good case in point. Topics in his collection of vlogs include practical tutorials like 'bindings for beginners' as well as more emotive vlogs about struggling with the bureaucracy of an inept healthcare system, gender dysphoria and intimacy with a partner, or being subjected to misgendering. But he also produces more lighthearted videos like the one billed 'trans guy gift ideas'. These may be tongue in cheek funny, but actually also contain a lot of practical information about what makes everyday life a little bit easier for a young trans guy. Lots of young trans vloggers recognise the particular practical and social pressures their peers face and one response to this are the DIY gender videos that aim to problem solve things like how to get hold of a binder or how to make your own packer if you can't order online because you don't have a credit card, or you are too young to order online. The main interest of this article is however primarily the meaning making processes of the audience, in this case the young people who engage with the videos. My empirical findings suggest their engagement with these videos is complex, and involves careful and multimodal viewing strategies. The young people interviewed for this project talked about how they paid close attention to every detail in the vloggers' videos, including body language, style, voice, fashion, demeanour and physical changes, and 
commented on how useful they found this information located outside the narrative content. The audio-visuality of the video medium is a really important component. There is a lot of meaning to be drawn from the micro level of gender performativity that is coded into the audio - such as pitch of voice and manner of speaking - as well as in the visual self-representation. As Raun (2015, 369) points out, 'transitioning technologies, body, camera and visuality intersect' in different ways in these videos. In other words, they offer a complex and haptic sensation of embodiment that is less about simple representation and more about being in space, and as such constitutes an often-overlooked form of cultural production of key importance to LGBTQ youth audiences. Further ethnographic research would be needed to better understand the depth and complexity of their engagement with the audio-visual codes as significantly rich in meaning.

To conclude, it can be noted that the trans youth in this study take what can be described as a pragmatic approach as consumers of vlogs. They look to the vloggers for information that is often of a quite practical nature and that they might apply to their own lives. Beyond Raun’s (2016) work on trans vlogging that notes how the genre involves sharing of practical advice, such as for example medical information, this is still an area of the care structures communities build that is under-theorized. I argue that for young people in particular this specific area of help on how to make one’s everyday life liveable is very important. This is not to say that discourses of transitioning or organised political advocacy are not of interest to young people, they absolutely are, but for many teenagers in particular, these bigger questions may have less immediate bearing on their everyday lives and the vloggers' peer support on how to handle difficult teachers or parents, or advice on how to keep safe in public spaces 
or where to shop for certain items of clothing should also be acknowledged as important.

\section{Concluding remarks}

For many the internet connection is a crucial lifeline, as testified by several participants in this research and supported by research on resilience in LGBTQ youth (Craig et al, 2015). But young people are not always in control of if, how and where they access online media. Despite such sometimes real felt mechanisms of disempowerment and the issue of mainstream culture's excluding of or misrepresentation of trans lives, trans youth digital culture is growing in volume and range of expression. This project has found that trans youth find a diverse range of online culture meaningful to their understanding of themselves and their world, this includes important virtual counterpublics and SNS that are conducive to countercultural community formation and non normative gender discourse. Whilst longstanding structures for LGBTQ cultural and identity formation still dominate, this research gives an indication of new languages, aesthetic forms and publics forming in online digital culture.

Drawing on Kitchin and Dodge’s (2011) work, we may argue that software has the potential to aid the transformation of the teenager's bedroom into a civic and community space; a space for peer education, activism and enculturation. Indeed many vloggers cited by the Transformers such as Jake Edwards, Alex Bertie, Upper Case Chase, Jessica Tiffany and Isabella Bunny Bennett illustrate this in different ways. However, participation online is inevitably shaped by the platforms' fundamental structure which is imprinted with the particular socio-economic, raced and cis-gendered logic of the culture they predominantly serve. Young LGBTQ 
people are very aware of how the cis-gendered, straight and white norms that surround them also prevail online and will have to be negotiated, as this article has shown.

Avoiding simplistic utopian or dystopian conclusions, I hope to have foregrounded the mundane yet persistent resistance trans youth in this study offer in their questioning of mainstream paradigms and in their creation of a new symbolic language for activism through DIY vlogs, widgets, cat gifs, fan mashups and unicorns farting rainbows in the face of homo- and transphobia.

\section{Acknowledgements}

I would like to thank Allsorts staff and especially Freiya Benson for their guidance and comments on an earlier draft of this article, and the Transformers participants for generously sharing their thoughts with me.

\section{Notes on contributor}

Dr Olu Jenzen is Principal Lecturer in Media Studies at the University of Brighton. Her research ranges over different contemporary themes in Media Studies and Critical Theory with a particular interest in the politics of aesthetic form and popular culture as it intersects with debates of gender and sexualities, activism, marginalized communities, heritage, and social media. Current projects include an AHRC funded project on the Aesthetics of Protest and a University of Brighton funded social engagement award about the activist lives of young LGBTQ people.

\section{References}

Baker, Paul, and Amanda Potts. 2013. “"Why Do White People Have Thin Lips?” Google and the Perpetuation of Stereotypes via Auto-complete Search Forms.' Critical Discourse Studies, 10(2): 187-204.

boyd, danah. 2011. Social Network Sites as Networked Publics. In A Networked Self: Identity, Community and Culture on Social Network Sites, edited by Zizi Papacharissi, 39-58. New York: Routledge. 
Browne, Kath, and Leela Bakshi. 2013. Ordinary in Brighton?: LGBT, Activisms and the City. London: Routledge.

Capuzza, Jamie Colette. 2016. 'Improvements Still Needed for Transgender Coverage.’ Newspaper Research Journal, 37(1): 82-94.

Carah, Nicholas, and Amy Dobson. 2016. ‘Algorithmic Hotness: Young Women’s “Promotion” and “Reconnaissance” Work via Social Media Body Images.' Social Media + Society, 2(4): 1-10.

Cavalcante, Andre. 2013. The Struggle for the Ordinary: Media Culture, Transgender Audiences and the Achievement of Everyday Life (Doctoral Dissertation). Accessed 1 August 2016. http://hdl.handle.net/2027.42/100102.

Cavalcante, Andre. 2016. “ “I Did It All Online:” Transgender Identity and the Management of Everyday Life.' Critical Studies in Media Communication, 33(1): 109-122.

Cheney-Lippold, John. 2011. 'A New Algorithmic Identity Soft Biopolitics and the Modulation of Control Theory.' Culture \& Society, 28(6): 164-181.

Craig, Shelley L., McInroy, Lauren, McCready, Lance T., and Ramona Alaggia. 2015. 'Media: A Catalyst for Resilience in Lesbian, Gay, Bisexual, Transgender, and Queer Youth.’ Journal of LGBT Youth, 12(3): 254-275. Dame, Avery. 2016. 'Making a Name for Yourself: Tagging as Transgender Ontological Practice on Tumblr.' Critical Studies in Media Communication, 33(1): 23-37.

DeNardis, Laura, and Andrea M. Hackl. 2016. 'Internet Control Points as LGBT Rights Mediation.’ Information, Communication \& Society, 19(6): 753-770.

Dhaenens, Frederik. 2012. 'Queer Cuttings on YouTube: Re-editing Soap Operas as a Form of Fan-produced Queer Resistance.' European Journal of Cultural Studies, 15(4), 442-456.

Driver, Susan. 2007. Queer Girls and Popular Culture: Reading, Resisting, and Creating Media. New York: Peter Lang.

Fink, Marty and Quinn Miller. 2014. 'Trans Media Moments: Tumblr, 2011-2013.' Television \& New Media, 15(7): 611-626.

Gillespie, Tarleton. 2010. 'The politics of “platforms.”' New Media \& Society, 12(3): 347-364.

GLSEN, CiPHR, \& CCRC (2013). Out Online: The Experiences of Lesbian, Gay, Bisexual and Transgender Youth on the Internet. New York: GLSEN. 
Grossman, Arnold H., and Anthony R. D’Augelli. 2006. ‘Transgender Youth: Invisible and Vulnerable.' Journal of Homosexuality, 51(1): 111-128.

Hart, Matthew. 2015. 'Youth Intimacy on Tumblr: A Pilot Study.' Young, 23(3): 193208.

Horak, Laura. 2014. 'Trans on YouTube Intimacy, Visibility, Temporality.' TSQ:

Transgender Studies Quarterly, 1(4): 572-585.

House of Commons. 2016. Transgender Equality Report, 14 January. London: The Stationery Office Limited.

Jenzen, Olu, and Irmi Karl. 2014. 'Make, Share, Care: Social Media and LGBTQ Youth Engagement.' Ada: A Journal of Gender, New Media, and Technology, No.5. http://adanewmedia.org/2014/07/issue5-jenzenkarl/

Jones, Tiffany, and Lynne Hillier. 2013. 'Comparing Trans-Spectrum and Same-sexAttracted Youth in Australia: Increased Risks, Increased Activisms.' Journal of LGBT Youth, 10(4): 287-307.

Kitchin, Rob, and Martin Dodge. 2011. Code/Space. Software and Everyday Life. Cambridge, MA: MIT Press.

Kosenko, Kami A., Bond, Bradley J., and Ryan J Hurley. 2016. 'An Exploration Into the Uses and Gratifications of Media for Transgender Individuals.' Psychology of Popular Media Culture. No pagination.

Laukkanen, M. 2007. 'Young Queers Online: The Limits and Possibilities of Nonheterosexual Self-representation in Online Conversations.’ In Queer Online: Media Technology and Sexuality, edited by Kate O’Riordan and David J Philips, 81-100. New York: Peter Lang.

Lenhart, Amanda. 2015. 'Teens, Social Media and Technology: Overview.' Pew Research Center, Internet Science and Tech. Accessed 1 September 2016. http://www.pewinternet.org/2015/04/09/teens-social-media-technology-2015/

Lennon, Erica, and Brian Mistler. 2014. 'Cisgenderism.' TSQ: Transgender Studies Quarterly, 1(1-2): 63-64.

McInroy, Lauren B., and Shelley L Craig. 2015. 'Transgender Representation in Offline and Online Media: LGBTQ Youth Perspectives.' Journal of Human Behaviour in the Social Environment, 25(6): 606-617.

Nakamura, Lisa. 2013. 'Glitch Racism: Networks as Actors within Vernacular Internet Theory.' Culture Digitally: Examining Contemporary Cultural 
Production. Accessed 1 July 2017 http://culturedigitally.org/2013/12/glitchracism-networks-as-actors-within-vernacular-internet-theory/

Oakley, Abigail. 2016. 'Disturbing Hegemonic Discourse: Nonbinary Gender and Sexual Orientation Labeling on Tumblr.' Social Media + Society, 2(3): 1-12. Pullen, Christopher, ed. 2014. Queer Youth and Media Cultures. Basingstoke: Palgrave Macmillan.

Raun, Tobias. 2015. 'Video Blogging as a Vehicle of Transformation: Exploring the Intersection between Trans Identity and Information Technology.' International Journal of Cultural Studies, 18(3): 365-378.

Raun, Tobias. 2016. Out Online: Trans Self-Representation and Community Building on YouTube. London: Routledge

Renninger, Bryce J. 2014. 'Where I Can Be Myself... where I Can Speak my Mind: Networked Counterpublics in a Polymedia Environment.' New Media \& Society, 17(9): $1513-1529$.

Roth, Yoel. 2014. 'Locating the "Scruff Guy": Theorizing Body and Space in Gay Geosocial Media.’ International Journal of Communication, 8: 2113-2133.

Ryan, Caitlan, and Ian Rivers. 2003. 'Lesbian, Gay, Bisexual and Transgender Youth: Victimization and its Correlates in the USA and UK.' Culture, Health \& Sexuality, 5(2): 103-19.

Shapiro, Eve. 2004. 'Transcending Barriers: Transgender Organizing on the Internet.' Journal of Gay and Lesbian Social Services, 16(3-4): 165-179.

Tompkins, Avery. 2014. ‘Asterisk.’ TSQ: Transgender Studies Quarterly, 1(1-2): 2627.

Transgender Europe. 2009-2016. Trans Murder Monitoring. Accessed 1 November 2016 http://transrespect.org/en/research/trans-murder-monitoring/

Trans Media Watch. 2010. How Transgender People Experience the Media. Accessed 21 September 2016 transmediawatch.org

Warner, Michael. 2002. Publics and Counterpublics. Cambridge, MA: Zone Books. 